\title{
Aspectos Nutricionais de Lactentes Participantes no Programa Mãe-Bebê
}

\author{
Caroline D’Azevedo Sica , ${ }^{1}$ Janaína Simon Adams, ${ }^{2}$ Ilse Maria Kunzler, \\ Simone de Paula, Daiana Picoloto, Lisara Carneiro Schacker, \\ Maristela Cássia de Oliveira Peixoto, Carmen Esther Rieth
}

\section{RESUMO}

Nas últimas décadas o Aleitamento Materno Exclusivo (AME) no Brasil apresentou tendência ascendente, cujos principais ganhos foram observados entre 1986 e 2006, seguido de uma estabilização em 2013, que foi de 36,6\%. A presente pesquisa avaliou aspectos nutricionais de lactentes de 0 a 6 meses atendidos no Programa Mãe-Bebê. Trata-se de um estudo quantitativo e retrospectivo. Foi utilizado banco de dados de puérperas e lactentes atendidos no Programa Mãe-Bebê, no período de março de 2016 até junho de 2018. Foram incluídos lactentes que receberam AME e/ou misto até o sexto mês de idade. A classificação do peso ao nascer e classificação do estado nutricional foi descrito por meio das curvas de crescimento de peso para o comprimento $(\mathrm{P} / \mathrm{C})$ e comprimento para idade (C/I), conforme preconizado pela OMS. A amostra foi composta por 79 lactentes e $34,2 \%$ foram amamentados exclusivamente. Ao correlacionar P/C com duração do AME não houve diferença significativa, contudo, 26,6\% das crianças em AME foram classificadas eutróficos quando avaliado P/C aos 6 meses de idade, e 34,2\% das crianças em AME até 180 dias estavam com comprimento adequado para a idade. Quando avaliamos o P/C observamos que 27,8\% nasceram com risco para sobrepeso e após 6 meses de idade estavam classificadas em obesidade. Podemos concluir com este estudo que crianças amamentadas exclusivamente ao seio no primeiro semestre de vida apresentaram estado nutricional de eutrofia, evidenciando que o leite materno é o alimento ideal para o crescimento adequado nos seis primeiros meses de vida.

Palavras-chave: Aleitamento materno; estado nutricional; lactentes.

\section{NUTRITIONAL ASPECTS OF INFANT PARTICIPANTS IN A MOTHER-BABY PROGRAM}

\section{ABSTRACT}

In recent decades, exclusive breastfeeding (EBF) in Brazil has shown an upward trend, whose main gains were observed between 1986 and 2006, following a stabilization in 2013 that was 36.6\%. The present study evaluated nutritional aspects of infants aged 0 to 6 months attended in the Mother-Baby Program. It is a quantitative and retrospective study. A database of mothers and infants treated in the Mother-Baby Program from March 2016 to June 2018 was used. Infants who received EBF and / or mixed until the sixth month of age were included. Birth weight classification and nutritional status classification were described using the weight growth curves for length $(\mathrm{P} / \mathrm{C})$ and length for age $(\mathrm{C} / \mathrm{I})$, as recommended by WHO. The sample consisted of 79 infants and $34.2 \%$ were exclusively breastfed. When correlating P/C with EBF duration, there was no significant difference, however, $26.6 \%$ of EBF children were classified as eutrophic when assessed P / C at 6 months of age, and 34.2\% of EBF children up to 180 days were of adequate length for age. When assessing $\mathrm{P} / \mathrm{C}$, we observed that $27.8 \%$ were born at risk for overweight and after 06 months of age were classified as obese. We can conclude from this study that exclusively breastfed children in the first semester of life had nutritional status of eutrophic, showing that breast milk is the ideal food for proper growth in the first six months of life.

Keywords: Breastfeeding; nutritional status; infants.

RECEBIDO EM: 10/2/2020

MODIFICAÇÕES SOLICITADAS EM: 26/4/2021

ACEITO EM: 8/7/2021

\footnotetext{
${ }^{1}$ Autora correspondente. Universidade Feevale - Conselho Superior, Curso de Nutrição. Campus II - ERS 239, Vila Nova. Novo Hamburgo/RS, Brasil. CEP 90620-000. http://lattes.cnpq.br/1786136144042917. http:// orcid.org/0000-0002-3410-7151. carolinesica@gmail.com

2 Universidade Feevale. Novo Hamburgo/RS, Brasil.
} 


\section{INTRODUÇÃO}

Já é sabido que o aleitamento materno, além de ser um ato de amor, é um meio de nutrição eficaz, completo e que fornece diversos nutrientes importantes para o desenvolvimento saudável das crianças pequenas. ${ }^{1}$ Nas últimas três décadas, o Aleitamento Materno Exclusivo (AME) no Brasil apresentou tendência ascendente, cujos principais ganhos foram observados entre 1986 e 2006, seguido de uma estabilização em 2013, que foi de $36,6 \%$. Conforme os resultados preliminares do Estudo Nacional de Alimentação e Nutrição Infantil (Enani), que ocorreu entre fevereiro de 2019 e março de 2020, que avaliou 14.584 crianças com menos de 5 anos de vida, observou-se que $53 \%$ das crianças brasileiras seguem sendo amamentadas até o seu primeiro ano de idade e $45,7 \%$ das crianças menores de seis meses são amamentadas exclusivamente, e nas menores de quatro meses esse índice é de $60 \%{ }^{2}$ Mesmo que o estudo tenha apontado valores positivos, ainda assim há crianças que são desmamadas antes da idade de 2 anos, ou nem recebem aleitamento materno exclusivo até os seus primeiros 6 meses conforme recomendado pelo Ministério da Saúde. ${ }^{1}$

O Aleitamento Materno (AM) de forma exclusiva deve ser o primeiro alimento de um recém-nascido. Sua superioridade no que diz respeito a nutrientes para as funções biológicas o faz ser considerado o melhor alimento para crianças, por ter papel importante na proteção imunológica contra doenças infecciosas, na adequação nutricional e no desenvolvimento afetivo e psicológico, e é recomendado por especialistas do mundo inteiro., ${ }^{3,4}$

O AM é de fundamental importância para a saúde do bebê, visto que é produzido especificamente para atender suas necessidades nutricionais e proporcionar desenvolvimento e crescimento adequados. Ademais, sua relevância se dá em fortalecer o sistema imunológico, reduzindo os riscos de morbidade e mortalidade por infecções, como diarreia e infecções respiratórias. ${ }^{5,6}$

O crescimento no primeiro ano de vida é um processo complexo com mudanças rápidas, sendo afetado direta ou indiretamente por diversos fatores, como sexo, raça, o peso ao nascer, condições de saúde da criança e tipo de alimentação. Esses fatores são influenciados por condições socioeconômicas, culturais e biológicas. Diante de sua natureza multicausal, portanto, o crescimento infantil deve ser avaliado levando em consideração fatores relacionados com a sua etiologia e o seu desenvolvimento. ${ }^{8}$

Identificar os fatores que influenciam a adesão e o sucesso do AM permite promover ajustes nas práticas de promoção e incentivo ao aleitamento. Neste contexto, o objetivo do presente estudo foi avaliar aspectos nutricionais de lactentes de 0 a 6 meses de idade atendidos em um projeto de extensão da Universidade Feevale.

\section{MÉTODOS}

Trata-se de um estudo quantitativo, descritivo, retrospectivo, com delineamento transversal. Foi utilizado o banco de dados de puérperas e lactentes atendidos em um projeto de extensão da Universidade Feevale, denominado Progra-

Editora Unijuí - Revista Contexto \& Saúde - ISSN 2176-7114 - v. 21, n. 44, out./dez. 2021 
ma Mãe e Bebê, que está vinculado a uma Estratégia de Saúde da Família (ESF) de um município do Vale do Rio dos Sinos - RS, que tem como objetivo atuar na promoção da saúde da mulher tanto no período gestacional quanto no puerpério, assim como para o lactente desde o nascimento até o primeiro ano de idade, mediante ações educativas e interdisciplinares a fim de incentivar a prática do AM e hábitos alimentares saudáveis, entre outras ações.

A amostra foi composta por lactentes de 0 a 6 meses de idade atendidos no Programa Mãe e Bebê. Foram utilizados como critérios de inclusão todos os questionários que estivessem preenchidos de forma completa e os lactentes que receberam Aleitamento Materno Exclusivo (AME) e/ou misto até o sexto mês de idade. Como critérios de exclusão utilizou-se lactentes que não receberam AM em nenhum momento, prematuros e mães que não assinaram o Termo de Consentimento Livre e Esclarecido (TCLE) e o TCLE para menores de idade.

O banco de dados foi elaborado a partir do preenchimento dos questionários aplicados no programa de extensão, no período de março de 2016 até junho de 2018. O referido banco de dados foi alimentado com informações como idade, altura, peso e Índice de Massa Corporal (IMC) pré-gestacional da puérpera. Além disso, esses dois últimos dados foram aferidos na última semana de gestação, obtendo assim o ganho de peso total gestacional; tipo de parto, sexo do bebê, comprimento e peso ao nascimento e aos seis meses de idade, duração do AME em dias, motivo do desmame e idade do início da alimentação complementar em meses. A classificação do peso ao nascer foi dada conforme preconizada pela Organização Mundial da Saúde (OMS), baixo peso (<2.500g), peso insuficiente (2.500-2.999g), peso adequado (3.000-3.999g) e excesso de peso (>4000g). ${ }^{9}$

Os pesos e comprimentos utilizados foram obtidos por meio da caderneta da criança, que contém esse acompanhamento de forma sistemática na ESF de referência. A classificação do estado nutricional dos lactentes foi descrita por meio das curvas de crescimento de peso para o comprimento (P/C), com diagnóstico para magreza, risco para baixo peso, eutrofia, risco para sobrepeso, sobrepeso e obesidade, e comprimento para idade (C/I), com diagnóstico de muito baixa estatura, baixa estatura e estatura adequada para idade, conforme preconizado pela OMS (2007). ${ }^{10}$

Os dados foram tabulados no software Statistical Package for Social Sciences (SPSS) versão 22.0 e as análises de frequência efetuadas no mesmo programa. Variáveis de distribuição normal foram apresentadas na forma de média e desvio padrão. As variáveis categóricas foram descritas como frequências absolutas e relativas. As análises entre as variáveis quantitativas foram avaliadas pelo teste qui-quadrado. 0 nível de significância estatística adotado foi de $5 \%$ ( $p$ $<0,05)$.

O Programa de Extensão Mãe e Bebê foi submetido ao Comitê de Ética em Pesquisa (CEP) da Universidade Feevale e Núcleo Municipal de Educação em Saúde Coletiva (Numesc) do município de Novo Hamburgo, sendo aprovado no CEP sob o número de parecer 2.340.689 em outubro de 2017, respeitando a Resolução número 466/12 do Conselho Nacional de Saúde. 


\section{RESULTADOS}

A amostra deste estudo foi constituida por 79 lactentes, e as características gerais das nutrizes e lactentes são apresentadas na Tabela 1. A média de idade materna foi de $24,84 \pm 6,02$ anos. Com relação aos dados das nutrizes observamos que a média de peso pré-gestacional foi de $68,52 \pm 15,67 \mathrm{~kg}$, a média de IMC pré-gestacional foi de $27,35 \pm 6,56 \mathrm{~kg} / \mathrm{m}^{2}$, a média do peso no final da gestação foi de $80,20 \pm 15,42 \mathrm{~kg}$ e a média do IMC no final da gestação foi de $31,98 \pm 6,46 \mathrm{~kg} / \mathrm{m},{ }^{2}$ sendo observado um ganho de peso durante o período gestacional de $11,68 \pm 5,04 \mathrm{~kg}$. Os lactentes da nossa amostra nasceram com a média de idade gestacional de $39,23 \pm 1,09$ semanas, assim classificados como a termo, sendo 50,6\% do sexo masculino e $69,6 \%$ nascidos de parto normal. A média do peso ao nascer foi de $3.376,54 \pm 460,30$ gramas, a média do comprimento ao nascer foi de $48,78 \pm 2,11 \mathrm{~cm}$, já aos seis meses de idade a média do peso foi de 7.808,03 $\pm 1047,91$ gramas e 0 comprimento aos seis meses foi de $66,28 \pm 2,65 \mathrm{~cm}$. Na nossa amostra $34,2 \%$ dos lactentes foram amamentados exclusivamente ao seio até o sexto mês de vida e 46,8\% tiveram introduzida a alimentação complementar ao sexto mês de vida.

Tabela 1 - Caracterização das nutrizes e lactentes da amostra ( $\mathrm{N}=79)$

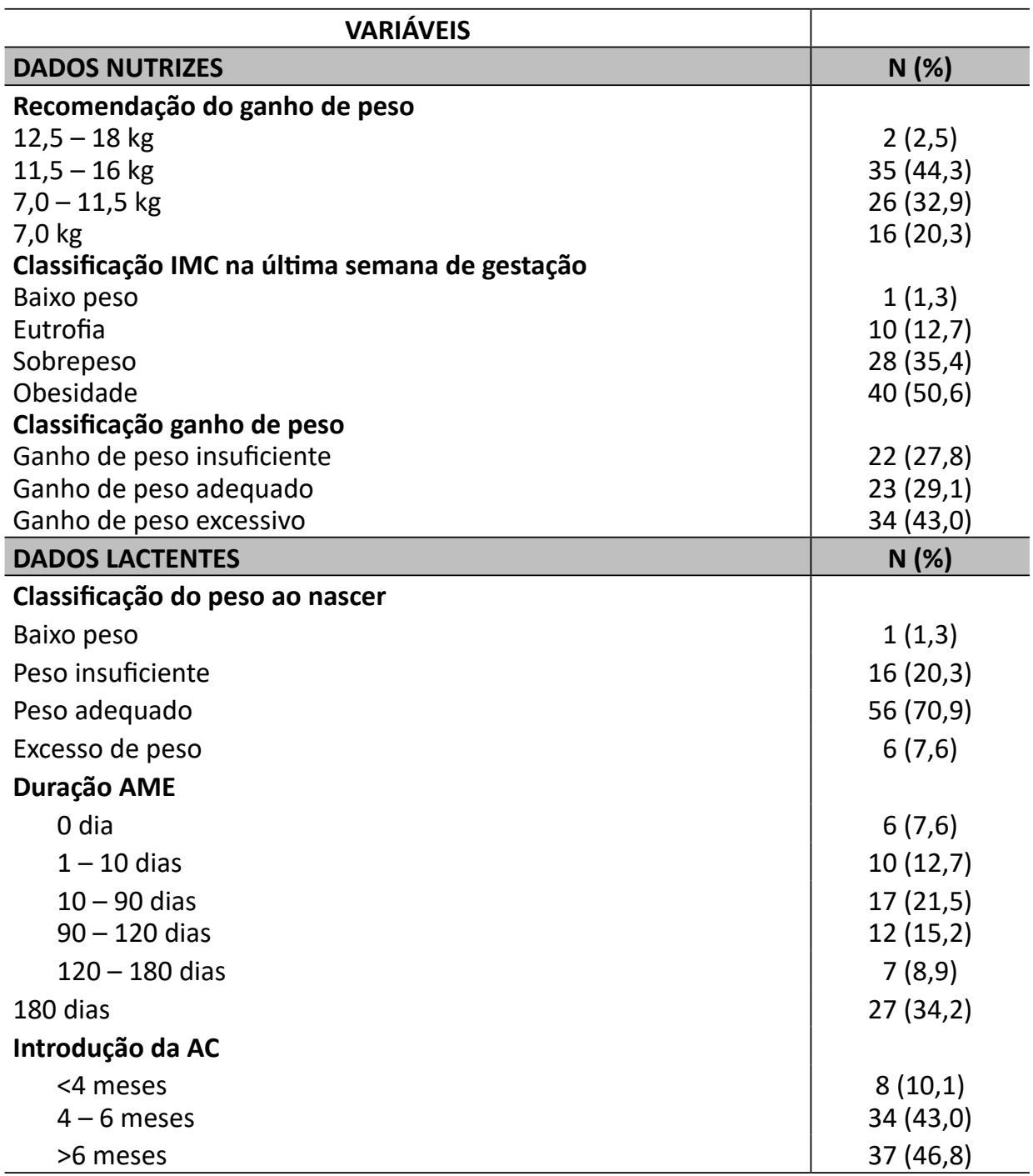

Kg: Quilograma; AC: Alimentação Complementar; AME: Aleitamento Materno Exclusivo; IMC: Índice de Massa Corporal. Fonte: Dados da pesquisa. 
Os diagnósticos nutricionais para Peso para Comprimento $(\mathrm{P} / \mathrm{C})$ e Comprimento para Idade (C/I) ao nascer e aos 6 meses de idade estão apresentados no Gráfico 1.

Gráfico 1 - Comparação do diagnóstico nutricional ao nascimento e aos 6 meses de idade da amostra $(\mathrm{N}=79)$

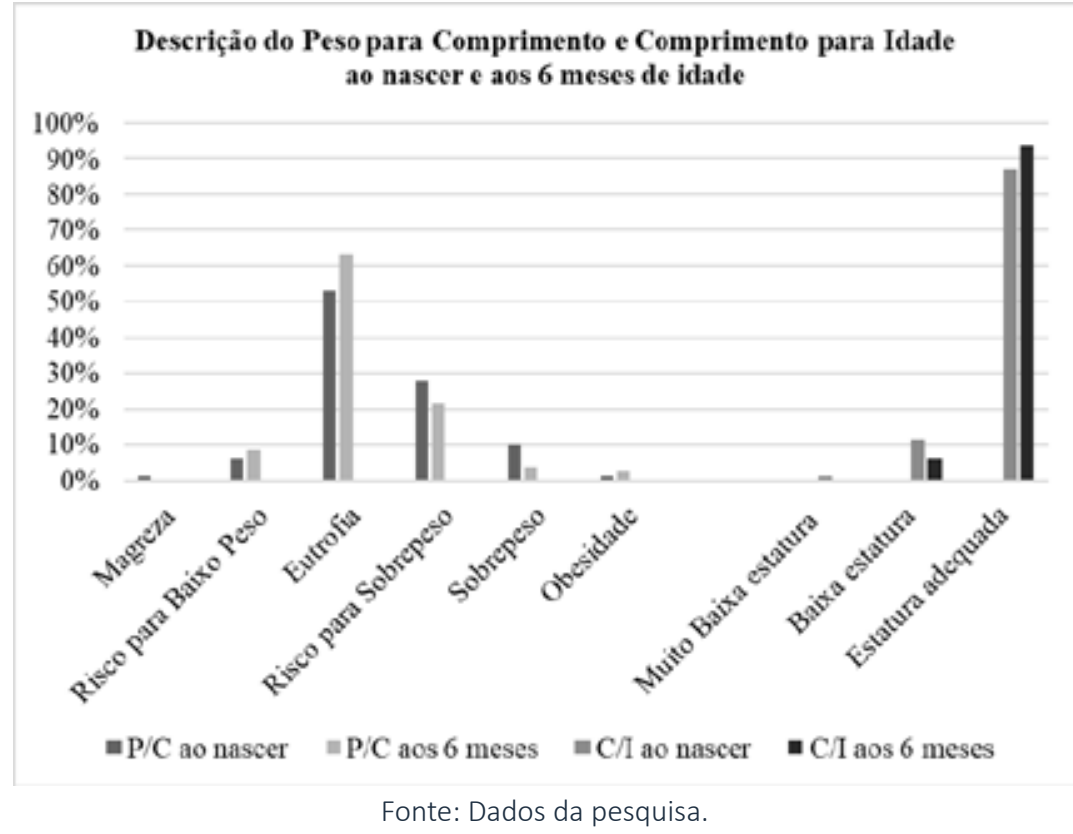

Na Tabela 2 podemos observar, quando comparamos $\mathrm{P} / \mathrm{C}$ com a duração do AME não houve diferença significativa apresentando um valor $p=0,095$, porém, 26,6\% das crianças em AME estão classificadas em eutrofia quando avaliado P/C aos 6 meses de idade, e 34,2\% das crianças amamentadas exclusivamente com leite materno até 180 dias estavam com a estatura adequada para a idade.

Tabela 2 - Análise do tempo de aleitamento materno exclusivo com o $\mathrm{P} / \mathrm{C}$ e $\mathrm{C} / \mathrm{I}$ aos seis meses de idade $(\mathrm{N}=79)$

TEMPO DE ALEITAMENTO MATERNO EXCLUSIVO

\begin{tabular}{|c|c|c|c|c|c|c|c|}
\hline \multirow{6}{*}{$\begin{array}{c}\mathrm{P} / \mathrm{C} \text { aos } \\
6 \text { meses } \\
\text { de idade } \\
-\mathrm{n}(\%)\end{array}$} & & $\begin{array}{c}0 \\
\text { dia }\end{array}$ & $\begin{array}{l}1-10 \\
\text { dias }\end{array}$ & $\begin{array}{c}10-90 \\
\text { dias }\end{array}$ & $\begin{array}{c}\text { 90-120 } \\
\text { dias }\end{array}$ & $\begin{array}{c}\text { 120-180 } \\
\text { dias }\end{array}$ & $\begin{array}{l}180 \\
\text { dias } \\
\end{array}$ \\
\hline & $\begin{array}{l}\text { Risco para } \\
\text { baixo peso }\end{array}$ & $1(1,3)$ & $1(1,3)$ & $4(5,1)$ & $1(1,3)$ & $0(-)$ & $0(-)$ \\
\hline & Eutrofia & $3(3,8)$ & $4(5,1)$ & $9(11,4)$ & $9(11,4)$ & $4(5,1)$ & $21(26,6)$ \\
\hline & $\begin{array}{l}\text { Risco para } \\
\text { sobrepeso }\end{array}$ & $0(-)$ & $4(5,1)$ & $4(5,1)$ & $2(2,5)$ & $2(2,5)$ & $5(6,3)$ \\
\hline & Sobrepeso & $1(1,3)$ & $1(1,3)$ & $0(-)$ & $0(-)$ & $0(-)$ & $1(1,3)$ \\
\hline & Obesidade & $1(1,3)$ & $0(-)$ & $0(-)$ & $0(-)$ & $1(1,3)$ & $0(-)$ \\
\hline \multirow{2}{*}{$\begin{array}{c}C / I \text { aos } \\
6 \text { meses } \\
\text { de idade } \\
-n(\%)\end{array}$} & $\begin{array}{l}\text { Baixo } \\
\text { comprimento } \\
\text { para idade }\end{array}$ & $1(1,3)$ & $0(-)$ & $3(3,8)$ & $1(1,3)$ & $0(-)$ & $0(-)$ \\
\hline & $\begin{array}{l}\text { Comprimento } \\
\text { adequada } \\
\text { para idade }\end{array}$ & $5(6,3)$ & $10(12,7)$ & $\begin{array}{c}14 \\
(17,7)\end{array}$ & $\begin{array}{c}11 \\
(13,9)\end{array}$ & $7(8,9)$ & $27(34,2)$ \\
\hline
\end{tabular}

P/C: Peso para comprimento; C/I: Comprimento para idade.

Fonte: Dados da pesquisa. 
Verificamos na Tabela 3 que 35,4\% da amostra que estava classificada em eutrofia ao nascer, mantiveram a mesma classificação após 6 meses de idade, porém 27,8\% que nasceram com risco para sobrepeso, após 6 meses de idade estavam classificadas em obesidade. Não houve diferença significativa quando comparamos as variáveis, apresentando um valor $p=0,539$.

Tabela 3 - Comparação do $\mathrm{P} / \mathrm{C}$ ao nascer e $\mathrm{P} / \mathrm{C}$ aos 6 meses de idade $(\mathrm{N}=79)$

\begin{tabular}{|c|c|c|c|c|c|c|}
\hline \multicolumn{7}{|c|}{$P / C$ aos 6 meses $-n(\%)$} \\
\hline \multirow{7}{*}{$\begin{array}{l}\mathrm{P} / \mathrm{C} \mathbf{R N} \\
\mathrm{n}(\%)\end{array}$} & & $\begin{array}{l}\text { Risco para } \\
\text { baixo peso }\end{array}$ & Eutrofia & $\begin{array}{l}\text { Risco para } \\
\text { sobrepeso }\end{array}$ & Sobrepeso & Obesidade \\
\hline & Magreza & $0(-)$ & $1(1,3)$ & $0(-)$ & $0(-)$ & $0(-)$ \\
\hline & $\begin{array}{l}\text { Risco para } \\
\text { baixo peso }\end{array}$ & $1(1,3)$ & $2(2,2)$ & $2(2,2)$ & $0(-)$ & $0(-)$ \\
\hline & Eutrofia & $5(6,3)$ & $28(35,4)$ & $8(10,1)$ & $0(-)$ & $1(1,3)$ \\
\hline & $\begin{array}{l}\text { Risco para } \\
\text { sobrepeso }\end{array}$ & $0(-)$ & $15(19,0)$ & $3(3,8)$ & $1(1,3)$ & $22(27,8)$ \\
\hline & Sobrepeso & $1(1,3)$ & $3(3,8)$ & $4(5,1)$ & $0(-)$ & $0(-)$ \\
\hline & Obesidade & $0(-)$ & $1(1,3)$ & $0(-)$ & $0(-)$ & $0(-)$ \\
\hline
\end{tabular}

$\mathrm{P} / \mathrm{C}$ : Peso para comprimento; RN: recém-nascido.

Fonte: Fonte: Dados da pesquisa.

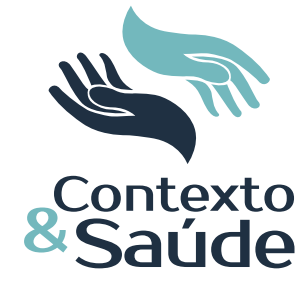

\section{DISCUSSÃO}

O Aleitamento Materno (AM) é uma prática essencial para a promoção da saúde física, psíquica e mental do bebê e traz inúmeros benefícios para a mãe, principalmente no pós-parto, além de promover o vínculo do binômio mãe-bebê. A hipótese inicial era que a prática do AM influenciaria positivamente no ganho ponderal de lactentes de 0 a 6 meses de idade. Com relação aos lactentes, a maioria nasceu eutrófico; já quando analisada pelo $\mathrm{P} / \mathrm{C}$, essa classificação permaneceu, sendo a maioria quando passados seis meses de vida. Essa informação pode ser relacionada ao fato de que um terço da população estudada recebeu AME até 180 dias de vida.

A média de idade materna deste estudo foi semelhante com a pesquisa realizada pelo Ministério da Saúde, em que mulheres entre 20 e 35 anos apresentaram maior frequência de AME $(44,4 \%)$, porém no tocante AM, identificou-se maior prevalência (82,5\%) em mães mais jovens ( $<20$ anos). ${ }^{11}$ Simões et al. (2015), ${ }^{12}$ em um estudo realizado sobre as dificuldades envolvidas na amamentação, também concluíram que a idade materna tem papel relevante no AM, pois mulheres com mais idade apresentam mais dificuldade em amamentar seus fiIhos, influenciando na manutenção da prática do aleitamento. Além de que, muIheres mais jovens têm mais acesso à informação de forma mais fácil no que se refere aos benefícios e vantagens do AM, diferentemente de estudo de Gigante et al. (2000), ${ }^{13}$ em que os achados demonstraram maior prevalência de AME aos seis meses conforme aumentava a idade da mãe.

Em relação ao tipo de parto, mais da metade das mães deste estudo tiveram parto normal. Alves et al. (2013) observaram que o parto cesáreo é fator de risco para a amamentação exclusiva, visto que diminuiu em $16 \%$ a prevalência 
desta, achado esse que vai ao encontro deste estudo. Alguns estudos concluíram que a via de parto é determinante para o sucesso da amamentação. Segundo eles, mulheres que têm parto cesáreo referem dor e desconforto após a cirurgia, dificultando as primeiras mamadas, a maneira de posicionar o bebê corretamente, podendo interferir na disposição da mãe em amamentar, além da demora da descida do leite, deixando o bebê suscetível à introdução de fórmulas lácteas de forma precoce, podendo ocorrer o desmame no primeiro mês de vida. ${ }^{14,15,16} \mathrm{~A}$ pesquisa de Lima et al. (2003) ${ }^{17}$ revelou que o percentual de crianças amamentadas exclusivamente era significativamente maior nas que nasciam de parto normal, com duração média de AME de 212,1 dias, enquanto as que nasceram de parto cesáreo alcançaram média de AME de 169,5 dias.

A amamentação é essencial para a saúde do binômio, o LM é capaz de suprir todas as necessidades nutricionais, imunológicas e psicológicas do bebê, diminuindo morbidade e mortalidade infantil. ${ }^{18}$ É o melhor alimento a ser ofertado até os 6 meses de idade da criança, visto que oferece energia e todos os nutrientes necessários para o crescimento do lactente..$^{19} \mathrm{~A}$ prevalência de AME da nossa população aos 6 meses ficou aquém das recomendações da OMS, bem como dos resultados encontrados no último inquérito sobre a prevalência de AM nas capitais brasileiras e Distrito Federal. Conforme o inquérito supracitado, a prevalência de AME para crianças menores de 6 meses foi de $41 \%$ e 38,2\%, no Brasil e em Porto Alegre respectivamente. Estudo realizado com mães adolescentes em Porto Alegre/RS, todavia, apresentou baixos níveis de AME aos 6 meses de idade $(13,8 \%)^{5}$. Segundo a Sociedade Brasileira de Pediatria (2017), ${ }^{20}$ os inquéritos nacionais realizados até o ano de 2008 demonstram uma prevalência de 52,1\% de AM em menores de 24 meses e de $36,6 \%$ de AME, constatada na última Pesquisa Nacional de Saúde, dado esse considerado insatisfatório pela OMS.

Em estudo realizado com uma amostra considerável da população brasileira, com crianças nascidas a partir de 1981, observou-se que em média 90\% delas são amamentadas inicialmente, o que dura em média 90 dias, tempo esse considerado curto para um país em desenvolvimento. Já o AME correspondia a apenas 6\% até os 2 meses de idade. ${ }^{21}$ Horta et al. (1996) ${ }^{22}$ constataram que em Pelotas a duração mediana do aleitamento materno, para as crianças menores de 12 meses, aumentou de 3,1 meses em 1982 para 4 meses em 1993. Aos 6 meses, $29 \%$ das crianças estavam sendo amamentadas em 1982, contra $38 \%$ em 1993, não fugindo muito do resultado da nossa pesquisa, passadas mais de duas décadas. Já em estudo recente de Oliveira et al. (2017),, ${ }^{23}$ verificou-se grande progresso nos achados sobre a amamentação no Brasil nas últimas quatro décadas. Houve aumento na duração do AM, passando de 2,5 meses em 1975 para 11,3 meses em 2008 um aumento de 14 vezes na prevalência do AME. Além disso, esse estudo inovou documentando que os fatores de risco modificáveis para a prática do aleitamento materno também mudaram nesse período.

A literatura traz inúmeros estudos sobre ganho ponderal de lactentes segundo o tipo de aleitamento materno que estes receberam, mas os resultados são controversos e seus autores recomendam mais pesquisas sobre o tema. No Brasil, porém, há poucos estudos a respeito. Há estudos sobre padrão de alimentação e crescimento, porém quando definida a faixa etária e tipo de alimentação,

Editora Unijuí - Revista Contexto \& Saúde - ISSN 2176-7114 - v. 21, n. 44, out./dez. 2021 
a tendência é que o número diminua, ficando menor ainda quando se trata de AME até o sexto mês de vida da criança. ${ }^{24}$

Os resultados deste estudo mostraram que as crianças sob AME apresentaram estado nutricional satisfatório durante os seis primeiros meses de vida. Esses dados vão ao encontro com o que a literatura preconiza a respeito da importância do AME nesse período como forma de prevenção da desnutrição e da obesidade. Barros (1981), ${ }^{25}$ em trabalho comparando leite humano e leite artificial e o efeito no ganho ponderal, demonstrou que a mediana de ganho ponderal era de $28 \mathrm{~g} / \mathrm{dia}$, no primeiro trimestre, e de $23 \mathrm{~g} / \mathrm{d}$, no segundo trimestre para os meninos que recebiam leite artificial, e de 22,4 e $20 \mathrm{~g} / \mathrm{d}$, respectivamente, para as meninas alimentadas com leite artificial. Para os lactentes que recebiam leite materno, esses valores foram, respectivamente, $31 \mathrm{~g} / \mathrm{d}$ e $19 \mathrm{~g} / \mathrm{d}$ para os meninos e $26,6 \mathrm{~g} / \mathrm{d}$ e $18,5 \mathrm{~g} / \mathrm{d}$ para as meninas. Houve ganho ponderal mediano semestral de $4.280 \mathrm{~g}$ para os meninos e $3.640 \mathrm{~g}$ para as meninas com aleitamento artificial e 4.435 e $4.080 \mathrm{~g}$, respectivamente, com aleitamento materno, ficando evidente o efeito protetor do aleitamento materno em relação ao crescimento que se manteve durante todo o primeiro semestre de vida.

Assim como nesta pesquisa, em um estudo de Barros et al. $(2008)^{26}$ também não foi observada diferença estatisticamente significante em relação ao ganho ponderal aos 6 meses de idade em relação ao AME, tampouco os índices E/I e P/E. Minossi et al. (2013), ${ }^{27}$ ao correlacionarem o tipo de AM pregresso com o estado nutricional de pré-escolares em idade de 1 a 6 anos de duas escolas particulares de Porto Alegre/RS, perceberam que a amostra que recebeu um aleitamento materno misto encontrava-se com maiores taxas de sobrepeso e obesidade. Em estudo de Barros e Victora (1998), ${ }^{24}$ foi possível constatar que crianças amamentadas exclusivamente ao seio apresentam crescimento mais rápido quando comparadas àquelas alimentadas com fórmula, nos dois primeiros meses de vida, mas posteriormente havia uma inversão em relação ao ganho ponderal.

Augusto et al. $(2007)^{8}$ chegaram à conclusão de que o crescimento nos primeiros 6 meses de vida é determinado por diversos fatores, mas que o AME proporciona um ganho ponderal adequado. Ainda no tocante ao ganho ponderal, em estudo recente de coorte de Fonseca et al. (2017), ${ }^{28}$ no qual se avaliou os determinantes do crescimento de crianças de até 6 meses de idade, observou-se maior mediana de crescimento nas crianças amamentadas exclusivamente no primeiro e segundo mês, diferentemente das alimentadas com fórmula infantil e das que já haviam introduzido água na alimentação, em que houve menor mediana de crescimento. Jaldin et al. $(2013)^{29}$ avaliaram o crescimento infantil no primeiro semestre de vida comparado com as referências National Center for Health Statistics (NCHS) e o padrão WHO/2006 em crianças amamentadas exclusivamente com leite materno e constataram que o crescimento ponderal assemelhou-se ao padrão WHO; já o crescimento linear mostrou-se compatível com os dois parâmetros. As crianças do estudo também tiveram maior ganho de peso nos dois primeiros meses de vida ocorrendo desaceleração até os 6 meses, porém ainda estando satisfatório o ganho ponderal.

Uma limitação do estudo é o tamanho da amostra com número parcialmente reduzido, devido ao grupo limitar-se a um bairro de uma cidade. Outra

Editora Unijuí - Revista Contexto \& Saúde - ISSN 2176-7114 - v. 21, n. 44, out./dez. 2021 
limitação foi a ausência de informações em alguns prontuários, o que impossibilitou que fossem utilizadas todas as participantes do projeto para o estudo em questão. Sugere-se que sejam feitos mais estudos como este, pois o assunto é de grande utilidade para a saúde pública, uma vez que se refere à importância da relação do tempo de aleitamento materno e o ganho ponderal dos lactentes até o sexto mês de vida. $O$ presente estudo também permite aos profissionais da saúde reconhecer quais os problemas reais na fase da amamentação e incentivo ao mesmo, facilitando a promoção em saúde materno-infantil.

Conclui-se que crianças amamentadas exclusivamente ao seio no primeiro semestre de vida apresentaram estado nutricional de eutrofia, ficando evidente ser o leite materno o alimento ideal para o crescimento adequado nos 6 primeiros meses de vida. Dado seus inúmeros benefícios no âmbito biológico e psicossocial tanto para a criança quanto para a mãe, a prática da amamentação exclusiva deve ser orientada e incentivada junto as mães desde a gestação. É imprescindível que conheçamos os fatores que favorecem e interferem no AME para que haja um planejamento de ações que beneficiem as famílias e comunidade de modo que os benefícios dessa prática possam ser plenamente usufruídos pelo binômio mãe/bebê.

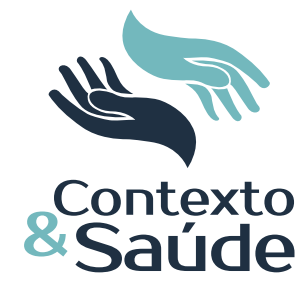

\section{REFERÊNCIAS}

${ }^{1}$ Brasil. Ministério da Saúde. Cadernos de atenção básica no 23. Saúde da criança: aleitamento materno e alimentação complementar. Brasília; 2015

2 Brasil. UNA-SUS. Pesquisa inédita revela que índices de amamentação cresceram no Brasil. Ago. 2020.

${ }^{3}$ Venancio SI, Escuder MML, Saldiva SRD, Giugliani ERJ. Breastfeeding practice in the Brazilian capital cities and the Federal District: current status and advances. J Pediatric. 2010;86(4):317-324.

${ }^{4}$ Muniz MD. Benefícios do aleitamento materno para a puérpera e o neonato: a atuação da equipe de saúde da família. Universidade Federal de Minas Gerais. Faculdade de Medicina. Núcleo de Educação em Saúde Coletiva. Formiga; 2010.

${ }^{5}$ Nunes LM. Importância do aleitamento materno na atualidade. Boletim Científico de Pediatria. 2015;4(3):55-8.

${ }^{6}$ Victora CG; Barros AJD; França GVA; Bahl RR; Nigel C.; Horton S; Krasevec J; Murch S; Sankar MJ; Walker N. Amamentação no século 21: epidemiologia, mecanismos e efeitos ao longo da vida. The Lancet, Brasília. 2016;387:1-24. Tradutor Garcia LP e França GVA de.

${ }^{7}$ Alves ALN, Oliveira MIC de, Moraes JR de. Iniciativa Unidade Básica Amiga da Amamentação e sua relação com o aleitamento materno exclusivo. [Internet]. Revista de Saúde Pública. 2013 dez.;47(6):1130-1140.

${ }^{8}$ Augusto RA, Souza JMP. Crescimento de crianças em aleitamento materno exclusivo no primeiro semestre de vida. Revista Brasileira Crescimento e Desenvolvimento Humano. 2007;17(2):1-11.

${ }^{9}$ Puffer RR, Serrano C. Patterns of birth weight. Washington (DC): PAHO; 1987. (Scientific Publication, 504).

${ }^{10}$ WHO Multicentre Growth Reference Study Group. Assessment of differences in linear growth among populations in the WHO Multicentre Growth Reference Study. Acta Paediatr. 2006;450(Suppl):56-65.

${ }^{11}$ Brasil. Ministério da Saúde. Secretaria de Atenção à Saúde. Departamento de Ações Programáticas e Estratégicas. II Pesquisa de Prevalência de Aleitamento Materno nas 
Capitais Brasileiras e Distrito Federal. [Internet]. Brasília: Editora do Ministério da Saúde, 2009.

12 Simões IAR, Rennó G, Salomon ASC, Martins MCM, Sá RAD. Influência dos mitos e das crenças nas nutrizes quanto à amamentação em uma cidade do Vale do Paraíba. Rev Ciênc Saúde. 2015.

${ }^{13}$ Gigante DP, Victora CG, Barros FC. Nutrição materna e duração da amamentação em uma coorte de nascimento de Pelotas, RS. Rev Saúde Pública. 2000;34(3):259-65.

${ }^{14}$ Faleiros FTV, Trezza EMC, Carandina L. Aleitamento materno: fatores de influência na sua decisão e duração. Rev Nutr 2006;19(5):623-630.

${ }^{15}$ Margotti E, Epifanio M. Exclusive maternal breastfeeding and the Breastfeeding Self-efficacy Scale. Rev Rene. 2014 sep./oct. [citado 20 sep. 2016]; 15(5):771-9.

${ }^{16}$ Weiderpass E, Barros, FC, Victora CG, Tomasi E, Halpern R. Incidência e duração da amamentação conforme o tipo de parto: estudo longitudinal no Sul do Brasil. Rev Saúde Pública. 1998;32(3):225-231.

17 Lima TM, Osorio MM. Perfil e fatores associados ao aleitamento materno em crianças menores de 25 meses da Região Nordeste do Brasil. Rev Bras Saúde Matern Infant. 2003;3(3):305-14.

18 Santos AJAO; Bispo AJB; Cruz LD. Padrão de aleitamento e estado nutricional de crianças até os seis meses de idade. HU Revista, Juiz de Fora, 2016 jul./ago.;42(2):119-24.

19 . Fialho FA et al. Fatores associados ao desmame precoce no aleitamento materno. Rev Cuidarte. 2014;5(1):670-8.

20 SBP - Sociedade Brasileira de Pediatria. Guia Prático de Atualização. Departamento Científico de Aleitamento Materno. Uso de chupeta em crianças amamentadas: Prós e contras. 2017.

${ }^{21}$ Barros FC, Victora CG. Breastfeeding and diarrhea in Brazilian children. Demographic and Health Surveys Further Analysis Series. 1990 mar.;3.

22 Horta BL, Olinto MT, Victora CG, Barros FC, Guimarães PRV. Amamentação e padrões alimentares em crianças de duas coortes de base populacional no sul do Brasil: tendências e diferenciais. Cad Saúde Pública. 1996;12 Supl 1:43-8.

${ }^{23}$ Oliveira DS; Boccolini CS; Faerstein E; Verly Júnior E. Breastfee-ding duration and associated factors between 1960 and 2000. J Pediatr, Rio de Janeiro. 2017;93:130-5.

${ }^{24}$ Victora CG, Morris SS, Barros FC, Horta BL, Weiderpass E, Tomasi E. Breast-feeding and growth in Brazilian infants. AmJClin Nutr. 1998;67:452-8.

${ }^{25}$ Barros Filho AA. Crescimento, morbidade e leite materno. Estudo longitudinal no Município de Pradópolis. Ribeirão Preto [Tese de Doutorado] - Faculdade de Medicina de Ribeirão Preto da USP; 1981.

${ }^{26}$ Barros VO et al. Aleitamento materno e crescimento de lactentes atendidos pelo programa de saúde da família. Rev. Nutrire: Soc. Bras. Alim. Nutr. J. Brazilian Soc. Food Nutr., São Paulo. 2008;33(3):111-121.

27 Minossi, V. et al. Duração do aleitamento materno e o excesso de peso. Cinergis. 2013;14(1):11-18.

${ }^{28}$ Fonseca PCA, Carvalho CA, Ribeiro SAV, Nobre LN, Pessoa MC, Ribeiro AQ, Priore SE, Franseschini SCC. Determinantes da velocidade média de crescimento de crianças até seis meses de vida: um estudo de coorte. Ciência e Saúde Coletiva. 2017.

${ }^{29}$ Jaldin MGM, Pinheiro FS, Santos AM, Muniz NC. Crescimento infantil comparado com as referências NCHS e o padrão WHO/2006. Rev. Nutr. 2013;26(1):17-26. 